\title{
Recording of smoking status: a survey of general practice records of those with and without smoking-related disease
}

Andrew Wilson, Terjinder Manku-Scott and David Shepherd Department of General Practice and Primary Health Care, University of Leicester, UK

\begin{abstract}
Recording of smoking status in general practice was compared according to the presence or absence of four diseases in which smoking cessation is important (asthma, hypertension, ischaemic heart disease and diabetes). Five of seven practices in one locality of Leicester took part. Our aims were to discover whether patients with these diseases were more likely to have a record of smoking status in their general practice notes than patients without them, and to examine the accuracy of this record compared with results from a patient questionnaire. For all diseases included, the proportion of records containing data on smoking status was higher if the disease was present than if it was not. When adjusted for age and sex, differences in recording reached statistical significance for asthma, diabetes and ischaemic heart disease, but not for hypertension. Smoking rates, as estimated from both the GP record and the postal questionnaire, were lower for all disease groups, but for asthma this did not reach statistical significance. In all disease groups GP data overestimated smoking prevalence compared with the postal questionnaire. We conclude that practices targeted their recording of smoking to those with smoking-related conditions, but achieving higher and more accurate rates of recording in those with smoking-related diseases remains a priority.
\end{abstract}

Key words: ascertainment; primary care; smoking

\section{Introduction}

Recent guidelines have encouraged general practitioners and practice nurses to use the consultation to establish whether a patient smokes and to offer brief advice to smokers at least annually (West et al., 2000). They suggest that information on smoking is collected for all consulters, but general practitioners have been found to favour discussion of smoking when opportunities exist to relate such advice to a patient's medical condition (Coleman and Wilson, 2000). Prioritizing patients with smoking-related conditions may be an effective strategy as there is some evidence that patients who attribute their condition to smoking are more mot-

\footnotetext{
Address for correspondence: Dr A. Wilson, Department of General Practice and Primary Health Care, University of Leicester, Leicester General Hospital, Leicester, LE5 4PW, UK. Email: aw7@le.ac.uk
}

ivated than other smokers to stop (Walters and Coleman, 2002).

There is evidence from postconsultation questionnaires that patients consulting their general practitioners with smoking-related problems are more likely to report that smoking was discussed in the consultation than patients without smokingrelated problems (Coleman and Wilson, 1999), and from population surveys that patients with increased cardiovascular risk are more likely to report receiving advice to quit (Silagy et al., 1992). However self-report of smoking advice has been found to overestimate advice giving when compared with audiotaped consultations (Wilson and McDonald, 1994), probably because of socially desirable response bias.

Having accurate data on smoking in the medical record is an essential first step in any strategy to encourage smokers to quit. The only study we are aware of which examined the relationship of 
smoking recording with morbidity was done in Australia. This found recording rates in general practice were higher if a smoking-related disease was present (Dickinson et al., 1989).

In a study comparing general practice smoking data with patient questionnaire data reported elsewhere (Wilson et al., 2000), we tested whether the proportion of patients with a recent record of smoking status differed according to the presence of four common chronic conditions for which advice about smoking is an essential part of management. We also examined the accuracy of recorded smoking status against self-report on the postal questionnaire.

\section{Methods}

All seven practices in one part of Leicester, defined by the boundaries of three wards, were invited to take part and five agreed. These wards had high standardized mortality ratios for smoking-related diseases, and smoking was identified as a priority issue by the collaborative purchasing group of which they were part, although no specific interventions to reduce smoking had been implemented. Of the 32450 residents in this locality 22480 $(69.3 \%)$ were registered with a participating practice.

A sample was drawn from practice registers of individuals aged 15 to 74 years. Sampling was random, weighted by practice size, and stratified by sex and 10-year age bands. The sample size of 2500 was calculated for the primary aim of the study, which involved gaining an accurate $( \pm 2 \%)$ estimation of smoking prevalence (expected to be about 30\%).

The medical records of those sampled were examined for records of smoking status during the previous six years and for any record of asthma, high blood pressure, heart disease or diabetes. These diseases were chosen due to their high prevalence and consensus about diagnostic criteria, compared with, for example, chronic obstructive airways disease. The timeframe was chosen as target payments operating at the time required records of smoking to be no more than six years out of date (Health Departments of Great Britain, 1993). The last record of smoking was used to ascribe current smoking status. Inter-rater reliability estimates for attribution of smoking status from the
GP record were calculated for each practice based on examination of 50 records by the two data extractors (TMS and SB). Levels of agreement were very good, with kappa coefficients ranging from 0.74 to 0.94 (Landis and Koch, 1977).

The second source of information was a postal questionnaire, with up to two reminders, sent to the same sample. It included questions about current and past smoking history and whether the respondent suffered from asthma, high blood pressure, heart disease or diabetes. The questions about smoking were identical to those used in the Scottish Heart Health survey, which were found to correlate well with biochemical markers of smoking (Woodward et al., 1991; Woodward and Tunstall-Pedoe, 1992).

Data were analysed to test the null hypothesis that there were no differences in recording of smoking status between individuals with and without smoking-related disease. Odds ratios were calculated for a record of smoking in GP notes for those with the listed diseases compared with those who did not have the disease. Odds were then adjusted using logistic regression to control for age and sex, as these had previously been found to predict the presence of a record of smoking status. The same analysis was done to compare smoking rates (from the GP record and from the patient questionnaire, respectively) for those with and without smoking-related diseases. Finally, where data on an individual's smoking status were available from both sources, agreement was assessed using the kappa statistic (Landis and Koch, 1977).

The study was approved by Leicestershire Health Authority's ethics committee and conducted in 1995-6, three years after target payments were introduced in the UK to encourage general practitioners to record smoking habit (Health Departments of Great Britain, 1992).

\section{Results}

The final sample size was 2490, and in 2432 $(97.7 \%)$ the general practice records were available for scrutiny. Prevalence of diseases from the GP record were as follows: asthma 209 (8.6\%), hypertension $185(7.6 \%)$, ischaemic heart disease 88 $(3.6 \%)$, diabetes $70(2.9 \%)$. These prevalences agreed well with patients' self-report on the postal questionnaire (kappas 0.65, 0.67, 0.70, 0.85, 
respectively). Overall response rate to the postal questionnaire was 76.5\% (1906/2490). It was higher for those with hypertension $(89.2 \%)$, diabetes $(85.7 \%)$ and ischaemic heart disease $(79.5 \%)$ and similar for those with asthma (76.1\%). The GP record was available for scrutiny for 1843 questionnaire responders.

Overall, 1784 (73.4\%) GP records contained an entry of smoking status in the last six years. Table 1 shows recording rates of smoking status for those with and without the listed diseases, with crude odds ratios and adjustment for age and sex. Up to six cases were excluded for each condition because of uncertainty in extracting data from the medical records. In all cases, recording rates were higher if the disease was present, with adjusted odds reaching statistical significance for asthma and ischaemic heart disease, but not for hypertension or diabetes (although numbers were small).

Table 2 show rates of smoking for those with and without the listed diseases, with crude odds ratios and adjustment for age and sex, using data from the medical record (excluding cases with no record of smoking status) and postal questionnaire, respectively. Smoking rates reported from the postal questionnaire were lower than those from the medical records. Data from both sources show rates of smoking are lower for those with smokingrelated conditions, although for asthma the differences were small and not statistically significant.
Adjusted odds ratios were consistently lower using questionnaire data than from using data from the medical records.

Data from both questionnaire and GP records were available for 302 individuals with one or more of the selected diseases. Results are compared in Table 3. This shows good agreement (kappa 0.75), but $20(6.6 \%)$ individuals who denied smoking in the questionnaire were recorded in GP records as smokers, and $11(3.6 \%)$ who reported smoking were recorded as nonsmokers.

\section{Discussion}

Although necessarily a small scale study as it involved scrutiny of manual records, our results suggest that, as in Australia, (Dickinson et al., 1989) UK general practice records are more likely to record smoking status in those with smokingrelated conditions. Even so, more than $10 \%$ of patients with diabetes, hypertension or ischaemic heart disease had no record of smoking in the last six years, and more than $20 \%$ of those with asthma had no record.

The prevalence of smoking for all disease groups, assessed from GP records and postal questionnaire, was lower for those with the disease than those without, although for asthma the difference was small and not statistically significant. The

Table 1 Smoking recording rates in general practice records $(n=2432)$ by presence or absence of stated disease

\begin{tabular}{|c|c|c|c|c|}
\hline & $\begin{array}{l}\text { Number of } \\
\text { records examined }\end{array}$ & $\begin{array}{l}\text { Record of } \\
\text { smoking status }\end{array}$ & $\begin{array}{l}\text { Odds ratio } \\
(95 \% \mathrm{Cl})\end{array}$ & $\begin{array}{l}\text { Odds ratio adjusted } \\
\text { for age and sex } \\
(95 \% \mathrm{Cl})\end{array}$ \\
\hline \multicolumn{5}{|l|}{ Asthma } \\
\hline Present & 209 & $164(78.5 \%)$ & $1.36(0.96,1.91)$ & $1.54(1.08,2.21)$ \\
\hline Absent & 2217 & $1616(72.9 \%)$ & & \\
\hline Uncertain & 6 & & & \\
\hline \multicolumn{5}{|l|}{ Hypertension } \\
\hline Present & 185 & $153(82.7 \%)$ & $1.81(1.22,2.68)$ & $1.26(0.83,1.93)$ \\
\hline Absent & 2242 & $1626(72.5 \%)$ & & \\
\hline Uncertain & 5 & & & \\
\hline \multicolumn{5}{|c|}{ Ischaemic heart disease } \\
\hline Present & 88 & $77(87.5 \%)$ & $2.62(1.38,4.94)$ & $2.02(1.04,3.91)$ \\
\hline Absent & 2338 & $1702(72.8 \%)$ & & \\
\hline Uncertain & 6 & & & \\
\hline \multicolumn{5}{|l|}{ Diabetes } \\
\hline Present & 70 & $59(84.3 \%)$ & $1.98(1.03,3.79)$ & $1.61(0.08,3.22)$ \\
\hline Absent & 2358 & $1722(73.0 \%)$ & & \\
\hline Uncertain & 4 & & & \\
\hline
\end{tabular}


Table 2 Prevalence of smoking from GP records and patient questionnaire by presence or absence of stated disease

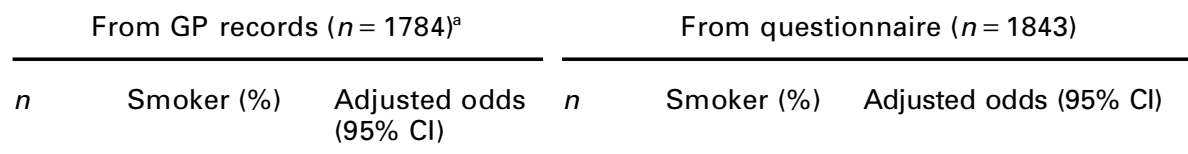

\begin{tabular}{|c|c|c|c|c|c|c|}
\hline \multicolumn{7}{|l|}{ Asthma } \\
\hline Present & 164 & $63(38.4)$ & \multirow[t]{2}{*}{$0.83(0.59,1.16)$} & 159 & $51(32.1)$ & \multirow[t]{2}{*}{$0.80(0.56,1.15)$} \\
\hline Absent & 1616 & $686(42.5)$ & & 1684 & $584(34.7)$ & \\
\hline Uncertain & 4 & & & & & \\
\hline \multicolumn{7}{|l|}{ Hypertension } \\
\hline Present & 153 & $37(24.2)$ & \multirow[t]{3}{*}{$0.43(0.28,0.64)$} & 165 & $29(17.6)$ & \multirow[t]{3}{*}{$0.30(0.25,0.58)$} \\
\hline Absent & 1626 & 712 (43.8) & & 1678 & $6.08(36.2)$ & \\
\hline Uncertain & 5 & & & & & \\
\hline \multicolumn{7}{|c|}{ Ischaemic heart disease } \\
\hline Present & 77 & $23(29.9)$ & \multirow[t]{2}{*}{$0.59(0.35,1.00)$} & 70 & $10(14.3)$ & \multirow[t]{2}{*}{$0.31(0.16,0.60)$} \\
\hline Absent & 1702 & $762(42.7)$ & & 1773 & $626(35.3)$ & \\
\hline Uncertain & 5 & & & & & \\
\hline \multicolumn{7}{|l|}{ Diabetes } \\
\hline Present & 59 & $14(23.7)$ & \multirow[t]{3}{*}{$0.45(0.25,0.84)$} & 60 & $10(16.7)$ & \multirow[t]{3}{*}{$0.33(0.16,0.68)$} \\
\hline Absent & 1722 & 736 (42.7) & & 1783 & $626(35.1)$ & \\
\hline Uncertain & 3 & & & & & \\
\hline
\end{tabular}

${ }^{a}$ Those with no smoking entry excluded from denominator.

Table 3 Contingency table for last smoking record in notes and current smoking status from questionnaire for the 302 individuals with data from both sources and having asthma, hypertension, ischaemic heart disease or diabetes

\begin{tabular}{llll}
\hline \multirow{2}{*}{ Notes } & \multicolumn{2}{c}{ Questionnaire } & Totals \\
\cline { 2 - 3 } & \multicolumn{2}{c}{ Smoker } & Nonsmoker \\
& \multicolumn{2}{l}{} \\
\hline Smoker & 69 & 20 & $89(29.5)$ \\
Nonsmoker & 11 & 202 & 213 \\
Totals & $80(26.5)$ & 302 \\
& & & kappa $=0.75$ \\
& & $P<0.001$ \\
\hline
\end{tabular}

higher prevalence estimates of smoking in all disease groups using GP data compared to questionnaire data could be due to three factors. First, GPs may be more likely to record the smoking status of smokers than nonsmokers, the so-called 'worst first' bias (Mant and Phillips, 1986). Secondly, although the questionnaire achieved a very high response rate, smokers were less likely to respond, as previously reported (Wilson et al., 2000). Thirdly we found evidence of some discrepancies between individuals' smoking status in the GP rec- ords compared with self-report, more often that the patient reported not smoking when they were recorded as a smoker. This is probably because the latest GP data were up to six years old, and demonstrates the need to document smoking behaviour more frequently in groups where smoking cessation is a priority.

The study was limited to recording of smoking status, and clearly not all discussions about smoking are documented in the GP record. Similarly, we were not able to measure whether advice about quitting was given, as few general practices record this in detail. Furthermore we cannot infer any causality between higher rates of recording of smoking status and lower prevalence rates of smoking.

In conclusion, we suggest that increasing the completeness and accuracy of smoking data for individuals with smoking-related problems should continue to be a priority in general practice.

\section{Acknowledgements}

We are grateful to participating general practitioners and their patients, to Jackie Cooney for help with data collection, to Sue Adams for statisti- 
cal advice and to Tim Coleman for his comments on an earlier draft. The study was funded by NHS Trent Research Scheme.

\section{References}

Coleman, T. and Wilson, A. 1999: Factors associated with provision of anti-smoking advice by general practitioners." British Journal of General Practice 49, 557-58.

Coleman, T. and Wilson, A. 2000: Anti-smoking advice from general practitioners: is a population-based approach to advice-giving feasible? British Journal of General Practice 50, 1001-1004.

Dickinson, J.A., Wiggers, J., Leeder, S.R. and Sanson-Fisher, R.W. 1989: General practitioners' detection of patients' smoking status. Medical Journal of Australia 150, 420-22.

Health Departments of Great Britain. 1993: General practice in the National Health Service: the 1993 contract. London: HMSO.

Landis, J.R. and Koch, G.G. 1977: The measurement of observer agreement for categorical data. Biometrics 33, 159-74.

Mant, D. and Phillips, A. 1986: Can the prevalence of disease risk factors be assessed from general practice records? British Medical Journal Clinical Research Ed 292, 102-104.
Silagy, C., Muir, J., Coulter, A., Thorogood, M., Yudkin, P. and Roe, L. 1992: Lifestyle advice in general practice: rates recalled by patients. British Medical Journal 305, 871-74.

Walters, N. and Coleman, T. 2002: Comparison of the smoking behaviour and attitudes of smokers who attribute respiratory symptoms to smoking with those who do not. British Journal of General Practice 52, 132-34.

West, R., McNeill, A. and Raw, M. 2000: Smoking cessation guidelines for health professionals: An update. Thorax 55, 987-99.

Wilson, A. and McDonald, P. 1994: Comparison of patient questionnaire, medical record, and audio tape in assessment of health promotion in general practice consultations. British Medical Journal 309, 1483-85.

Wilson, A., Manku-Scott, T., Shepherd, D. and Jones, B. 2000: A comparison of individual and population smoking data from a postal survey and general practice records. British Journal of General Practice 50, 465-68.

Woodward, M., Tunstall-Pedoe, H., Smith, W.C. and Tavendale, R. 1991: Smoking characteristics and inhalation biochemistry in the Scottish population. Journal of Clinical Epidemiology 44, 1405-10.

Woodward, M. and Tunstall-Pedoe, H. 1992: An iterative technique for identifying smoking deceivers with application to the Scottish Heart Health Study. Preventive Medicine 21, 88-97. 